\title{
A Perspective on Psychophysical Testing in Children
}

\author{
RUXANDRA SIRETEANU, MARIA FRONIUS, BIRGIT KATZ \\ Frankfurt, W. Germany
}

\begin{abstract}
Summary
In this paper, we show that the development of acuity in infancy is not due solely to foveal maturation, since there is a clear development of acuity in the peripheral visual field.

The development of peripheral acuity, and the naso-temporal asymmetry during early development reinforce the idea that the visual loss in adult strabismic amblyopes is not due to an arrest of development, but rather to the chronic interocular suppression of the deviated eye.

The Teller Acuity Card Test does not reliably detect strabismic amblyopia, and therefore should not be recommended as a screening test. However, the test is adequate for diagnosing visual losses in children with organic eye disorders and possibly anisometropia.
\end{abstract}

The preferential looking procedure is based on the fact that infants show a visual preference for a patterned stimulus, when this is presented simultaneously with a uniform surface of equal space-average luminance. ${ }^{1}$

By using grating stimuli of different spatial frequencies and the forced-choice response of an observer naive as to the side of presentation of the stimulus, it is possible to estimate the visual resolution of young infants ('forcedchoice preferential looking', FPL). ${ }^{2}$

In this paper, we present the results of two experiments based on the preferential looking procedure. In a first experiment, the development of visual acuity in the peripheral visual field was described for infants under one year of age. The main motivation for this study was to contribute to the understanding of the neural factors responsible for the rapid development of acuity in early infancy.

In a second experiment, infants and children at risk of amblyopia were tested with the newly developed Teller Acuity Cards (TAC). The results were compared with the results of established clinical methods (e.g. fixation preference, Landolt $\mathrm{C}$ acuity).

Results of both studies have been published in preliminary form.,

Experiment I: The development of peripheral visual acuity in infancy.

In a previous study, Sireteanu, Kellerer and Boergen ${ }^{5}$ tested the development of visual acuity at $10^{\circ}$ in the infants' peripheral visual field. They were primarily interested to learn whether the relatively advanced stage of morphological maturation of the infant peripheral retina is paralleled by functional maturity, reflected for instance by an adult-like level of visual acuity. The estimation of peripheral acuity was based on the infant's first saccade from a fixation stimulus towards a peripheral grating stimulus. In a second experiment, they tested the 'best acuity', using a method of forced-choice preferential looking and freeviewing conditions.

The results can be summarized as follows:

(1) there was a clear development of acuity in the peripheral visual field;

From: Max-Planck-Institute for Brain Research, Frankfurt, W. Germany.

Correspondence to: Ruxandra Sireteanu, Max-Planck Institute for Brain Research, Frankfurt, W. Germany. 
(2) peripheral acuity reached a plateau at about four months of age, while 'best acuity' continued to develop during the second half of the first year of life;

(3) at all ages tested, peripheral acuity was lower than the resolution evaluated with the same stimuli under free-viewing conditions. $^{5}$

The present investigation is an extension of this preliminary study. Our interests were twofold: (1) to learn whether the results obtained previously could be reproduced at another position in the visual field (20 degrees);(2) to test the development of visual acuity in monocular conditions. We were especially interested in the possible occurrence of naso-temporal asymmetries. This interest was prompted by the finding that in human infants, similar to young kittens, the detection of visual stimuli in the nasal visual field developed later than detection of objects presented in the temporal visual field. ${ }^{6,7}$

\section{Methods}

\section{Subjects}

Thirty-one infants between 17 days and 14 months of age were tested. Most of these infants were tested at more than one age, totalling 151 tests. Since longitudinally tested infants yielded similar results to the crosssectionally tested ones, our results are based on a combination of the two groups. Only fullterm infants with no developmental abnormalities and no ocular disorders were included in this study.

Six adult observers were tested binocularly and monocularly under identical experimental conditions. Three of these subjects were aware of the purpose of the experiments, the other three were not.

\section{Apparatus}

We used a modification of the forced-choice preferential-looking apparatus described by Sireteanu et al. ${ }^{5}$ It consisted of a large grey wooden screen containing two circular apertures of $15^{\circ}$ diameter, centered at $20^{\circ}$ from the midline. Four small red blinking lamps arranged around a peephole $(1 \mathrm{~cm}$ dia) served as a centering stimulus. Two of these lamps (either the vertical or horizontal ones) could be lit at a time.
At each trial, a square-wave grating of $80 \%$ contrast was projected from behind onto one aperture, and a blank stimulus (a uniform grey field of matched luminance) onto the other. The luminance of the stimuli was $75 \mathrm{~cd} / \mathrm{m}^{2}$.

For infants of all age groups, we used gratings of six different spatial frequencies: $0.6 \mathrm{c} /$ $\mathrm{deg}, 1.2 \mathrm{c} / \mathrm{deg}, 2.4 \mathrm{c} / \mathrm{dg}, 4.8 \mathrm{c} / \mathrm{deg}, 9.6 \mathrm{c} / \mathrm{deg}$ and $19.2 \mathrm{c} / \mathrm{deg}$. The gratings were purchased from Dr. R. Held (MIT, Cambridge, Massachusetts).

\section{Procedure}

The infant sat on its mother's lap, at a distance of $57 \mathrm{~cm}$ in front of the screen.

An adult observer, not knowing the side of presentation of the grating, looked at the infant's face on a video screen. A video camera was positioned behind the peephole. At the beginning of each trial, the observer attracted the infant's attention to the midline by blinking the fixation lamps and/or making calling noises. When she judged that the infant's gaze was centered on the midline, the blinking lights were extinguished, and a grating, paired with a blank stimulus, appeared on the screens. The sequence of presentation of the gratings was randomised.

The observer's task was to judge the direction of the first fixation from the centering stimulus toward a peripheral grating stimulus. For monocular testing, one of the infant's eyes was covered by an adhesive opaque occluder.

\section{Data analysis}

The subjects were divided into five age groups of 10-21 infants each: $0-4,4-6,6-8,8-10$ and 10-14 months. The data of all infants from every age group were collected to provide the psychometric curve of the respective age group. The curves were computer-fitted by cumulative normal ogives using a leastsquares method. The interpolated spatial frequency yielding a $70 \%$ correct response was taken as the acuity threshold of the age group.

\section{Results}

The development of binocular peripheral acuity

All groups of infants tested yielded orderly 
psychometric functions. The coarsest gratings elicited a high percentage of correct responses; for finer gratings, performance fell to chance.

There was a clear development of the binocular peripheral acuity in the first months of life. The resolution of gratings centered at $20^{\circ}$ increased from $1.2 \mathrm{c} / \mathrm{deg}$ to about $3 \mathrm{c} / \mathrm{deg}$ between 2.5 and 5 months. There was only a slight improvement in acuity to $5.4 \mathrm{c} / \mathrm{deg}$ at 11 months of age (Fig. 1, open circles).

\section{The development of monocular peripheral acuity}

The infants performed consistently less well under monocular than under binocular testing conditions. Some of the youngest infants (2-3 months old) and most of the oldest ones (10 months or more) refused to be tested with an eye patch. The upper asymptotes of the psychometric functions were lower, and the slopes more shallow.

Visual acuity developed from $1.1 \mathrm{c} / \mathrm{deg}$ at 3.5 months to about $4 \mathrm{c} / \mathrm{deg}$ at 10 months of age (Fig. 1, closed circles). Thus, monocular peripheral acuity was slightly, but consistently lower than binocular acuity throughout the tested period.
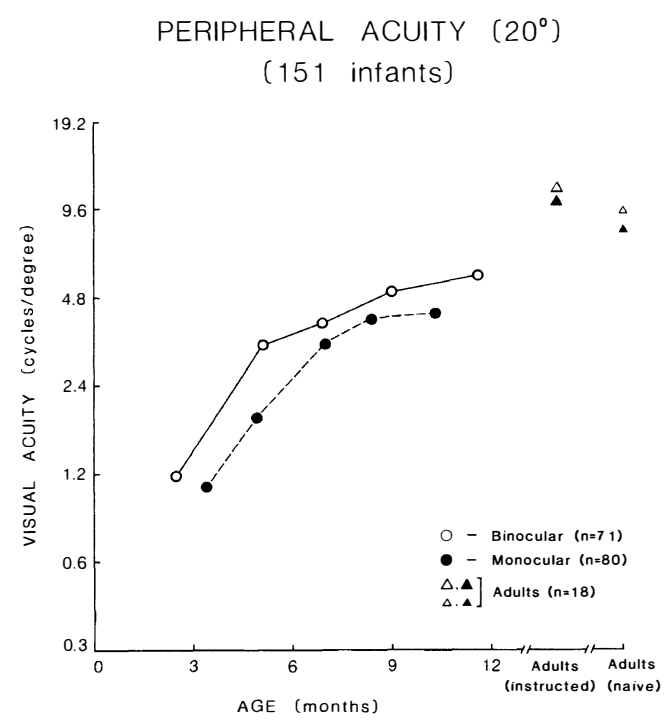

Fig. 1 Development of peripheral acuity in infants during the first year of life. Open symbols: binocular acuity; closed symbols: monocular acuity; circles: infants; large triangles: instructed adults; small triangles: naive adult observers.

\section{Peripheral acuity in adult observers}

The visual acuity of even the oldest infants tested (11-12 months old) remained significantly below that of normal adult observers tested under identical experimental conditions. This conclusion holds for both binocular and monocular testing conditions (see Fig. 1), regardless of whether the adults were aware of the purpose of the experiment (instructed) or not. Naive observers tended to give less consistent responses at lower spatial frequencies, and the slopes of their psychometric functions were more shallow, but their visual acuities were only slightly lower than those of the instructed observers (compare large and small triangles in Fig. 1).

\section{Naso-temporal asymmetry}

At all ages tested, acuity in the temporal visual field was higher than in the nasal field. Such an asymmetry was absent in adult observers tested under identical experimental conditions.

\section{Discussion}

The reduced visual function of the newborn infant was often attributed to the immaturity of the fovea: Indeed, the peripheral retina is morphologically well developed a few days after birth, while the central $5^{\circ}$ show a structural deficit, and might not be functional until about four months of age. ${ }^{8.9}$ Morphologically, the development of the fovea continues until at least four years of age. ${ }^{10}$

In the present study, we showed that there is a clear development of acuity in the peripheral visual field of human infants. We thus confirmed the results obtained by Sireteanu $e t$ $a l .{ }^{5}$ at another eccentricity. Unfortunately, the present results cannot be compared quantitatively with the previous ones, since there are small technical differences between the two studies: the first study was performed in a clinical setting, the second in a laboratory; the luminances of the test stimuli were slightly different; and the procedure of the first study was less automated than the present one.

Nevertheless, the two studies clearly show that the postnatal emergence and maturation of the fovea is not the only factor responsible for the development of acuity in early infancy.

Throughout the first year of life, acuity in 
the temporal field was higher than in the nasal visual field. This finding parallels the nasotemporal asymmetry in the detection of light spots reported by Maurer et al. ${ }^{7}$ and is consistent with anatomical reports that the nasal human retina (on which the temporal visual field is imaged) is more mature at birth than the temporal retina (corresponding to the nasal field). ${ }^{11}$

The occurrence of a naso-temporal asymmetry in the distribution of acuity in infancy brings some insight into the aetiology of amblyopia: In adult esotropic amblyopes, acuity is often more deeply affected in the near temporal than in the nasal visual field. ${ }^{25}$ This asymmetry is the opposite of that found in young infants. Therefore, it seems that the adult distribution of acuity in amblyopes is not the consequence of an arrest of development (as sometimes suggested) ${ }^{12}$ but rather the result of the chronic suppression of the deviated eye. Strabismic suppression selectively affects the nasal retina (temporal visual field) of this eye in esotropes. ${ }^{25,13}$

Experiment II: Clinical validation of the Teller Acuity Card Procedure

In spite of the obvious importance of a visual acuity test for preverbal children, the forced-choice preferential looking (FPL) procedure has been slow to be introduced into the clinical routine. Its main disadvantages are: the testing apparatus is complex and requires careful maintenance and recalibration; testing requires trained personnel and a tedious statistical analysis of the data; and the testing procedure is too lengthy to be carried out in the busy atmosphere of an eye clinic.

Based on the classical preferential looking procedures, a new test was created to measure the grating acuity of infants and toddlers, by using special acuity cards. ${ }^{* 14,15}$

These cards have been developed to overcome most of the above mentioned problems. The original stimuli consisted of cardboard cards containing a single vertical square-wave grating on one side and an equally-sized aperture of grey cardboard of matched average luminance ${ }^{14,15}$ or a high spatial frequency grating above the normal resolution ${ }^{16}$ on the other side; the rest of the card was uniformly grey. The testing procedure was much simpli- fied: instead of presenting the stimulus mechanically in random order, the cards were handheld, and presented in ascending order of spatial frequency; the observer was required to make a clincial judgement on the infant's ability to localize the grating. Visual acuity was defined as the highest spatial frequency the observer judged the infant to see. ${ }^{14-17}$

The acuity card procedure allows a rapid measurement of grating acuity. The comparison between acuity values obtained with the FPL procedure and those estimated with the acuity cards showed good agreement of the two procedures. ${ }^{18,19}$ In conclusion, the acuity card procedure was judged to be a useful clinical tool; several investigators suggested using it as a screening test for infants and preverbal children. ${ }^{20,21}$

Before recommending the TAC as a screening test or as an acuity test to be used in the clinical routine of ophthalmologists or pediatricians, it is important to examine whether the cards are sensitive to acuity losses due to ocular diseases and amblyopia. Since amblyopia is one of the most common causes for acuity loss in childhood, and effective treatment has to be started very early, a visual screening test for infants should be able to uncover amblyopia. It is well known that grating acuity is less affected by amblyopia (especially of strabismic origin) than recognition acuity. ${ }^{22,23}$ Since the TAC Test is based on the estimation of grating acuity, it is not clear whether amblyopia would be reliably diagnosed by this method. To examine this, we compared the grating acuity, estimated with the TAC Test, with the recognition acuity, tested with the Landolt $\mathrm{C}$ test, in amblyopic children that were old enough to complete both' tests. Additionally, in strabismic infants and children, the TAC Test was compared with the Fixation Preference Test.

A group of children with a variety of ocular disorders were also tested with the TAC Test. As a control group, normal children were included in this study.

\section{Methods}

The Teller Acuity Card Test

The TAC Test consisted of 15 cards, $25 \mathrm{~cm} \times$

\footnotetext{
* The acuity cards are commercially sold under the name of Teller Acuity Cards (TAC).
} 
$56 \mathrm{~cm}$ in size. Each card contained a $12.5 \times$ $12.5 \mathrm{~cm}$ black-and-white high-contrast square-wave grating on one side. The centres of the gratings were located $13.8 \mathrm{~cm}$ to the left or to the right of a central peephole. The space-average luminance of this test field was matched to the surrounding, so that the card appeared homogeneously grey if the grating was not resolved. The spatial frequency of the grating targets ranged from 0.32 to 38 cycles/cm in half-octave steps (an octave means a halving. or doubling of spatial frequency). At a test distance of $57 \mathrm{~cm}$, cycles/cm were equivalent to cycles/degree. Thirty cycles/degree corresponded to an acuity of 1.0 , respectively $20 / 20$ Snellen equivalent.

The test procedure was performed according to the handbook of the Teller Acuity Card Test:

During the test, the child was held (or stood) in front of a grey screen that contained a rectangular opening. An observer standing behind the screen attracted the child's attention towards this opening, and then held a test card up to it, without knowing the side of the grating target. During card presentation, the observer viewed the child's behaviour through the central peephole in the card. If the child showed a preference towards one side, this was taken as an indication that the presented grating was resolved. Before checking the actual grating location, the card was often turned 180 degrees, so that the grating appeared on the opposite side. If the patient's response changed appropriately, this proved his ability to resolve the grating. Then the actual grating location was checked. If upon the presentation of a grating, the child's behaviour did not show a side preference, this grating was judged not to be resolved.

The test cards were presented in ascending order, from coarse toward finer gratings. To save time, the coarser gratings were often presented in octave steps. It was switched to halfoctave steps when gratings close to the acuity limit were presented. Acuity was defined as the finest grating the observer judged the child to be able to see. The test distances were $40 \mathrm{~cm}$ for infants up to 10 months, $57 \mathrm{~cm}$ for children from 10 months to about three years old, and $80 \mathrm{~cm}$ for older children.

\section{Landolt C Test}

The Landolt $C$ optotypes were presented on cards containing rows of optotypes. The distance between the optotypes was at least 17.5 minutes of arc at a test distance of $80 \mathrm{~cm}$. The test we used is commercially available under the name of C-Test.

\section{Fixation Preference Test}

In strabismic infants and children, fixation preference was also tested. In children that showed spontaneously a strong fixation preference of one eye, we covered this eye while presenting a fixation object. If the squinting eye did not take up fixation under this condition, or took up fixation but fixation preference changed soon after uncovering the other eye, a monolateral strabismus was diagnosed. An alternating strabismus was diagnosed if no fixation preference was seen, but the left and the right eye fixated in turn. Some strabismics were able to alternate but commonly preferred one eye.

The Fixation Preference Test is commonly used in strabismic infants that are unable to pass visual acuity tests. If a fixation preference is present, amblyopia of the constantly squinting eye has to be suspected. On the other hand, amblyopia is unlikely in alternating strabismus with no fixation preference.

\section{Results}

Forty-nine normal children, 87 children at risk for amblyopia due to strabismus and/or anisometropia and 19 children with structural ocular disorders of one or both eyes were tested with the Teller Acuity Cards (TAC). In strabismic children, TAC-results were compared with the Fixation Preference Test. In older children the TAC Test was compared with a recognition test (Landolt C Test).

\section{Normal subjects}

Using the TAC, we first determined the monocular acuity of 49 normal subjects, two months to four years old (average 11.8 months). In 46 (93.9\%) children, both eyes could be tested, in three $(6.1 \%)$ only one eye. Of the monocular estimates, $84.2 \%$ were within the preliminary age norm offered with the TAC Test. Among the 46 subjects in which both eyes were tested, $30(65.2 \%)$ reached the 
same acuity in both eyes; $16(34.8 \%)$ had an interocular difference of one-half octave (corresponding to one test card). This difference was probably due to the inaccuracy of the test, and does not reflect a real interocular difference.

\section{Children with strabismus and/or anisometropia \\ Teller Acuity Card results}

Altogether, 87 children at risk for amblyopia, since they were strabismic and/or anisometropic, were tested monocularly with the TAC Test. Strabismus was diagnosed in 77 subjects; five of them also had an anisometropia. Ten subjects suffered from anisometropia only. All subjects were tested with the appropriate optical correction, except for two strabismics with equal refractive errors in both eyes. The mean age of the subjects was 5.8 years $(0.7$ to 13.8 years; see Fig. 2$)$. In $43(49.4 \%)$ of the children at risk for amblyopia, TAC acuity was equal in both eyes; $24(27.6 \%)$ subjects had an interocular difference of one-half octave. In $20(23.3 \%)$ children, an interocular difference of one octave or more was found

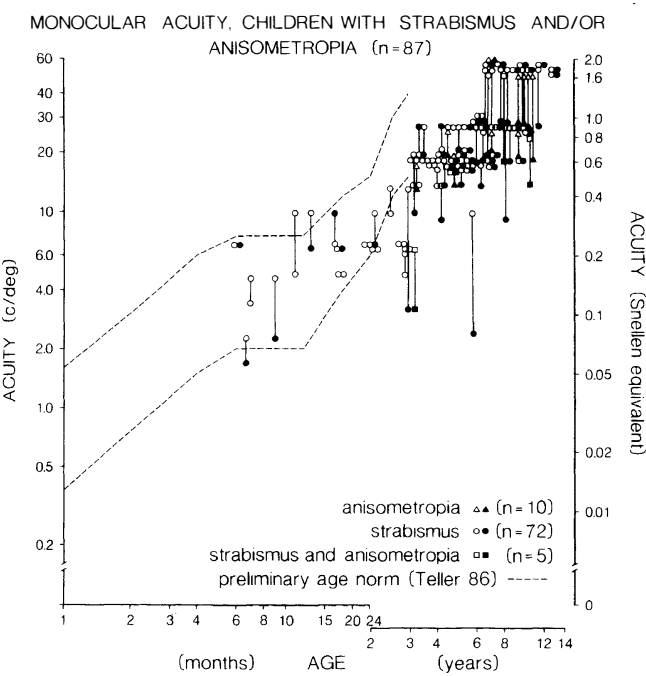

Fig 2. Monocular grating acuity in 87 strabismic and/or anisometropic children, evaluated with the Teller Acuity Card Test. Filled symbols are squinting eyes or, in anisometropic subjects, the eye with the higher refractive error. Open symbols symbolize the preferred, fixating eye in strabismics, or the eye with less refractive error in anisometropes. Pairs of two open symbols represent strabismics with alternating fixation, pairs of an open and a filled symbol represent monolateral strabismics and anisometropes. with the TAC. Thus, the group of children at risk for amblyopia showed more often an interocular difference than the normals $(50.6 \%$ vs. $34.8 \%)$. The largest interocular difference estimated in normals was one-half octave, while $23 \%$ of the subjects at risk for amblyopia had a more pronounced interocular difference.

\section{Comparison of grating acuity with fixation preference}

Of the 69 strabismic subjects that were probably amblyopic since they had a strong or slight fixation preference, only $16(23.2 \%)$ showed an interocular difference in grating acuity of one octave or more, thus higher than the interocular differences found in normals. Similar to the normals, the eight subjects with an alternating strabismus had at most one-half octave interocular difference in grating acuity.

\section{Comparison between Teller Acuity Cards and Landolt C Test}

A direct comparison of the two methods is shown in Fig. 3. The graph shows the relative acuity of the amblyopic eye (in percent of the acuity of the non-amblyopic eye), e.g. $50 \%$ means that the amblyopic eye reached half of the acuity of the non-amblyopic eye, $100 \%$ means that the same acuity was determined in both eyes. In $33(75 \%)$ of 44 subjects, the interocular acuity difference was larger with the Landolt Cs than with the TAC (data points below the $45^{\circ}$ line). Twenty-five $(75.8 \%)$ of these 33 children showed no interocular difference with the TAC, but very clear acuity losses with the Landolt $\mathrm{C}$ Test. However, there seemed to be no rule about how deep an amblyopia had to be, in order to be detectable with the TAC. On the contrary, from the children in whom the same depth of amblyopia was seen with the Landolt $C$ Test (e.g. $20 \%$ ), some showed a clear acuity loss with the TAC, but several had normal acuity values with this test (see Fig. 3).

Several children included in this drawing saw the $38 \mathrm{c} / \mathrm{cm}$ card with one or both eyes (see star-marked dots in Fig. 3); their acuity values might have been falsified by the edgeartifact of this card. Note, however, that even if these children are eliminated from the figure, the conclusion of a lack of correlation 


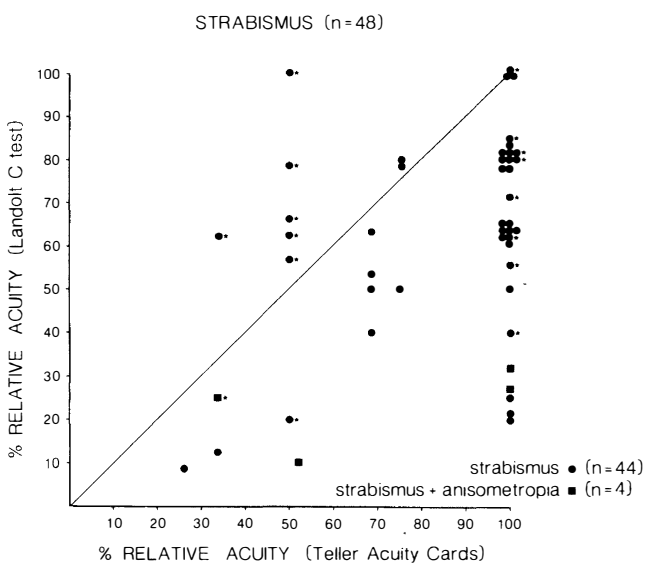

Fig. 3. Relative acuity of 48 amblyopic eyes (in percent of the acuity of the non-amblyopic eyes). This drawing allows a direct comparison between TAC and Landolt $C$, concerning the depth of amblyopia. Dots below the 45 degree line are cases in which amblyopia was more pronounced with Landolt Cs than with TAC. Dots above the 45 degree line represent children with a more pronounced interocular difference with $T A C$ than with Landolt Cs. The star-marked dots indicate children who saw the $38 \mathrm{c} / \mathrm{cm}$ card with one or both eyes; these values might have been falsified by the edgeartifact of this card (see also Robinson et al., 1988).

between the depth of amblyopia measured with TAC and with the Landolt $\mathrm{C}$ test holds.

The results in children with strabismus and anisometropia were very similar to those seen in strabismics. In all four subjects, the interocular acuity difference was less with the TAC Test than with the Landolt $\mathrm{C}$ Test.

The amblyopic as well as the nonamblyopic eyes of the nine non-strabismic subjects with anisometropia reached on the average nearly the same acuity with both procedures, but the acuity results of the amblyopic eyes showed a wider scatter. Three subjects showed a larger interocular difference with the Landolt $\mathrm{Cs}$ and four had more difference with the TAC. In two subjects, the interocular differences were nearly the same with both methods.

\section{Children with structural disorders of one or both eyes}

The grating acuity of 19 children 0.2 to 13.6 years old (average 5.2 years) with organic problems of one or both eyes was measured with the TAC (see Fig. 4).

Acuity loss based on ocular diseases (e.g., cataract, atrophy of the optic nerve and so on) affected the grating acuity measured with the Teller Cards more than strabismic amblyopia did. If only one eye was affected, its acuity was always lower than the acuity of the normal eye. If a subject had a bilateral ocular disease, the acuity estimates were usually below the age norm in both eyes (see Fig. 4).

There was a good agreement between TAC and Landolt $\mathrm{C}$ acuity.

\section{Conclusion}

The Teller Acuity Card Test does not detect reliably strabismic amblyopia. Since amblyopia is one of the most common causes of acuity loss in childhood, we do not recommend the Teller Acuity Card Test as a visual screening test. In preverbal strabismic children that are not able to pass recognition acuity tests, diagnosis and therapy control should continue to be based mainly on the classical clinical methods (e.g. Fixation Preference Test).

On the other hand, acuity losses due to structural ocular disorders are detectable with the Teller Acuity Card Test. In these cases, the Teller Acuity Card Test seems to be a useful adjunct to the clinical evaluation and therapy control.

These results show the necessity of investigations in order to develop a psychophysical test for children, which would capitalise on

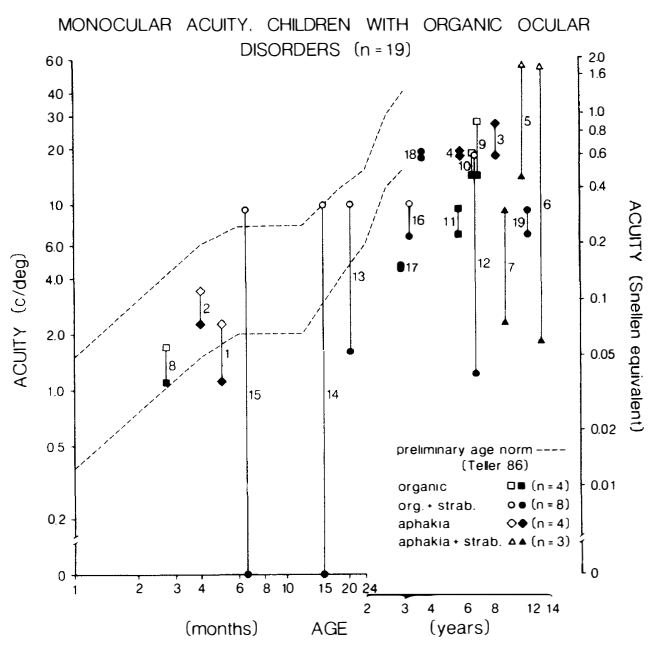

Fig. 4. Monocular TAC acuity of 19 subjects with structural eye diseases. Filled symbols: affected eye; open symbols: normal eyes. 
the advantages of the preferential looking technique, and at the same time be able to detect the more subtle losses of acuity due to an early strabismus.

The normal infants were tested at the Max-PlanckInstitute for Brain Research in Frankfurt.

We are grateful to Professor Wolf Singer for generous support and encouragement, to Sabine Alznauer for excellent technical assistance, to Dr. Dan $\mathrm{H}$. Constantinescu for help in processing the data, and to Gisela Knott for typing the manuscript. Dr. Barbara Engels participated in the early stages of this work. Thanks are due to our subjects and their parents for their patient collaboration.

Most of the observations on the children with clinical histories were done at the Eye Clinic of the University of Frankfurt. We are grateful to Prof. N. Stärk and to the orthoptists of the Eye Clinic for their friendly cooperation.

\section{References}

${ }^{1}$ Fantz RL: Pattern vision in young infants. Psychol Rec 1958, 8: 43-7.

${ }^{2}$ Teller DY, Morse R, Borton R, Regal D: Visual acuity for vertical and diagonal gratings in human infants. Vision Res 1974, 14: 1433-9.

${ }^{3}$ Sireteanu $\mathrm{R}$ and Fronius $\mathrm{M}$ : The development of peripheral visual acuity in human infants. In: Kaufmann H, Ed. Trans 16th Meeting European Strabismological Association, Giessen 1987, 221-9.

${ }^{4}$ Katz B and Sireteanu R: The Teller Acuity Card Test: a useful method for the clinical routine? Clin Vis Sci 1990, 5: 307-23.

${ }^{5}$ Sireteanu R, Kellerei R, Boergen K-P: The development of peripheral visual acuity in human infants. A preliminary study. Human Neurobiol 1984, 3: 81-5.

${ }^{6}$ Sireteanu R and Maurer D: The development of the kitten's visual field. Vision Res 1981, 22: 1105-11.

${ }^{7}$ Maurer D, Clarke AL, Lewis TL: The development of peripheral detection during infancy. Invest Ophthalmol Vis Sci 1986, 27 (3) suppl: 264.

${ }^{8}$ Mann I: The development of the human eye. London. British Medical Association, 1964.

${ }^{9}$ Abramov I, Gordon J, Hendrickson A, Hainline L, Dobson V, La Bossiere E: The retina of the newborn human infant. Science 1982, 217: 265-7.

${ }^{10}$ Yuodelis $\mathrm{C}$ and Hendrickson A: A qualitative and quantitative analysis of the human fovea during development. Vision Res 1986, 26: 847-55.

${ }^{11}$ Provis JM, Van Driel D, Billson FA, Russell P:
Development of the human retina: patterns of cell distribution and redistribution in the ganglion cell layer. J Comp Neurol 1985, 233: 429-51.

${ }^{12}$ Chavasse EB: Worth's squint. 7th edition. Philadelphia. Blakinston's Son, 1939.

${ }^{13}$ Sireteanu R: Human amblyopia: consequence of chronic interocular suppression. Human Neurobiol 1982, 1: 31-3.

${ }^{14}$ Dobson V, McDonald M, Teller DY: Visual acuity of infants and young children: Forced-choice preferential looking procedure. Am Orthopt J 1985, 35: 118-25.

${ }^{15}$ McDonald M, Dobson V, Sebris SL, Baitch L, Varner D, Teller DY: The acuity card procedure: a rapid test of infant acuity. Invest Ophthalmol Visual Sci 1985, 26: 1158-62.

${ }^{16}$ Teller DY, McDonald M, Preston K, Sebris SL, Dobson V: Assessment of visual acuity in infants and children: the acuity card procedure. Dev Med Child Neurol 1986, 28: 779-89.

${ }^{17}$ McDonald M, Sebris SL, Mohn G, Teller DY, Dobson V: Monocular acuity in normal infants: the acuity card procedure. Am J Optom Physiol Optics 1986, 63: 127-34.

${ }^{18}$ Preston KL, McDonald M, Sebris SL, Dobson V, Teller DY: Validation of the acuity card procedure for assessment of infants with ocular disorders. Ophthalmology 1987, 94: 644-53.

${ }^{19}$ Mohn G and van Hof-van Duin J: Rapid assessment of visual acuity in infants and children in a clinical setting, using acuity cards. Doc Ophthalmol Proc Series 1986, 45: 363-72.

${ }^{20}$ Mohn G, van Hof-van Duin J, Fetter WPF, De Groot L, Hage M: Acuity assessment in nonverbal infants and children: clinical experience with the acuity card procedure. Dev Med Child Neurol 1988, 30: 232-44.

${ }^{21}$ Sebris SL, Dobson V, McDonald M, Teller DY: Acuity cards for visual acuity assessments of infants and children in clinical settings. Clin Vision Sci 1987, 2: 45-58.

${ }^{22}$ Rentschler I, Hilz R, Brettel H: Spatial tuning properties in human amblyopia cannot explain the loss of optotype acuity. Behav Brain Res 1980, 1: 433-43.

${ }^{23}$ Mayer DL: Acuity of amblyopic children for small field gratings and recognition stimuli. Invest $O p h$ thalmol Vis Sci 1986, 27: 1148-53.

${ }^{24}$ Robinson J, Moseley MJ, Fielder AR: Grating acuity cards: spurious resolution and the 'edge artifact'. Clin Vision Sci 1988, 3: 285-88.

${ }^{25}$ Sireteanu R and Fronius M: Naso-temporal asymmetries in human ambloypia: consequence of long-term interocular suppression. Vision Res 1981, 21: 1055-63. 